\title{
Nox2 dependent redox-regulation of Akt and ERK1/2 to promote left ventricular hypertrophy in dietary obesity of mice
}

Article

Accepted Version

Creative Commons: Attribution-Noncommercial-No Derivative Works 4.0

Bhatti, S. N. and Li, J.-M. (2020) Nox2 dependent redoxregulation of Akt and ERK1/2 to promote left ventricular hypertrophy in dietary obesity of mice. Biochemical and Biophysical Research Communications, 528 (3). pp. 506-513. ISSN 0006-291X doi:

https://doi.org/10.1016/j.bbrc.2020.05.162 Available at https://centaur.reading.ac.uk/91056/

It is advisable to refer to the publisher's version if you intend to cite from the work. See Guidance on citing.

Published version at: http://dx.doi.org/10.1016/j.bbrc.2020.05.162

To link to this article DOI: http://dx.doi.org/10.1016/j.bbrc.2020.05.162

Publisher: Elsevier

All outputs in CentAUR are protected by Intellectual Property Rights law, including copyright law. Copyright and IPR is retained by the creators or other copyright holders. Terms and conditions for use of this material are defined in the End User Agreement. 


\section{CentAUR}

Central Archive at the University of Reading

Reading's research outputs online 
Journal: Biochemical and Biophysical Research Communications

DIO: $10.1016 /$ j.bbrc.2020.05.162

Nox2 dependent redox-regulation of Akt and ERK1/2 to promote left ventricular hypertrophy in dietary obesity of mice

\author{
Sunbal N. Bhatti* and Jian-Mei Li \\ School of Biological Sciences, University of Reading, UK
}

Short title: Nox2-mediated redox signaling in obese hearts

*Correspondence:

Miss Sunbal Naureen Bhatti

School of Biological Sciences

Harborne Building

University of Reading

Whiteknights

Reading, RG6 6AS

UK

Tel: 44 (0) 1183786466

Fax: $44(0) 1189310180$

Email: s.n.bhatti@pgr.reading.ac.uk

Professor Jian-Mei Li email: jian-mei.li@reading.ac.uk 


\section{Abstract}

Background: A Nox2 containing NADPH oxidase (Nox2) is involved in the global oxidative stress found in dietary obesity and metabolic disorders. However, the effects of high fat diet (HFD) on cardiac Nox 2 activation and signaling in left ventricular hypertrophy $(\mathrm{LVH})$ remain unknown.

Methods: Left ventricular (LV) tissues isolated from C57BL/6J wild-type (WT) and Nox2 knockout (Nox2KO) mice (11 months old, $n=6$ per group) after 4 months of HFD treatment were used. Cardiomyocyte sizes were measured digitally on LV cross-sections. The levels of cardiac reactive oxygen species (ROS) production was determined using lucigenin-chemiluminescence and in situ dihydroethidium (DHE) fluorescence. The levels of Nox subunit expression and redox signaling were examined by immunoblotting and immunofluorescence.

Results: In comparison to WT normal chow diet control hearts, WT HFD hearts had 1.8-fold increases in cardiomyocyte size, a sign of cardiac hypertrophy, and this was accompanied with $\geq 2$-fold increase in the levels of ROS production, Nox2 expression and the phosphorylation of Akt and ERK1/2. Increased ROS production measured in HFD heart homogenates was inhibited to control levels by Tiron (a cell membrane permeable $\mathrm{O}_{2}{ }^{\cdot-}$ scavenger), diphenyleneiodonium (DPI, a flavohaemoprotein inhibitor) and Nox 2 ds-tat (a Nox 2 assembly inhibitor). However, all of these abnormalities were significantly reduced or absent in Nox $2 \mathrm{KO}$ hearts under the same HFD.

Conclusions: Nox 2 activation in response to dietary obesity and metabolic disorders plays a key role in cardiac oxidative stress, aberrant redox signaling and cardiomyocyte hypertrophy. Knockout of Nox2 protects hearts from oxidative damage associated with obesity and metabolic disorders.

Key words: NADPH Oxidase; Redox-signaling; Cardiac hypertrophy; Akt; ERK1/2; Knockout mice 


\section{Introduction}

Obesity and diets high in fat are frequently associated with left ventricular hypertrophy (LVH) which is a principal cardiovascular complication that increases the risk of mortality [1]. As obesity is a chronic condition, the majority of cases had emerged in the middle-aged population. However, recently the rate of childhood and adolescent obesity soars [2], rendering investigation of the pathophysiology of obesity of even greater importance. Regular high dietary fat intake encourages health complications including hypertension, insulin resistance and dyslipidaemia which largely influence the structure and mechanics of the energy-demanding myocardium, instigating pathological adaptations comprising myocardial hypertrophy, remodelling and fibrosis [3].

Increasing attention is directed toward NADPH Oxidase 2 (Nox2), a principle generator of bioactive superoxide $\left(\mathrm{O}_{2}{ }^{--}\right)$, due to its influence in diet-induced oxidative damage in cardiovascular cell types [4]. Although crucial at low levels, excess Nox2-mediated $\mathrm{O}_{2}{ }^{--}$burden causes damage to macromolecules and organelles whilst impairing intracellular signaling events involving metabolic sensitivity [4]. Among the seven multisubunit Nox homologs of $65 \mathrm{kDa}$, Nox 2 is largely expressed in vascular cell types including endothelial cells, vascular smooth muscle cells and, principally, in cardiomyocytes mediating important roles in myocardial growth, remodelling and injury [5,6]. The heterodimeric flavohaemoprotein assembles at the plasma membrane after recruiting its subunits: Rac, p40 ${ }^{\text {phox }}, \mathrm{p} 47^{\text {phox }}$ and $\mathrm{p} 67^{\text {phox }}$ to generate the functional complex.

Multiple pro-hypertrophic stimuli are capable of activating Nox2 such as angiotensin II, mechanical stretch and tumour necrosis factor- $\alpha$ [5]. Accumulating evidence indicate that in obesogenic environments of chronic HFD intervention Nox2 expression is augmented in skeletal muscle [6] and aorta [7]. In the latter study Nox2-derived oxidative stress is reported to be essential for insulin response impairments, and often involves the redox-sensitive signaling intermediates Akt, ERK1/2 and p38MAPK [6-8]. However, this has not been investigated in myocardium. HFD feeding reduces insulin sensitivity as early as five days of intervention [9], associates with oxidative stress and promotes vascular damage involving cardiac remodelling, hypertrophy and apoptosis [10]. Aortic Nox2 overexpression occurs in HFD-fed ApoE knockout mice in unison with increased activation of both Akt and ERK1/2 and is associated with endothelial dysfunction [11]. Moreover, it has been reported that caloric restriction reverses endothelial oxidative damage in these conditions [12]. As obesity is an independent predictor of LVH, understanding the contribution of lipid-induced oxidative stress caused by Nox 2 in the manifestation of $\mathrm{LVH}$ is valuable. 
Recent promising evidence reveal the divergent roles of the pleiotropic Nox2 enzyme in endothelial cells compared with cardiomyocytes that, in pathological conditions, cause cell-type specific metabolic decline [13]. The importance of Nox signaling was reported in an experimental animal model of LVH, wherein ERK1/2 and p38MAPK activation mirrored Nox activity and were activated in response to pressure-overload [14]. Despite these new insights, there is insufficient evidence describing redox signaling activation and mechanism/s of HFD-induced Nox2 activation in the heart. Herein, utilising a mouse model of middle-age (11 months) HFD-induced metabolic syndrome, we demonstrate a pivotal role of myocardial HFD-induced Nox2 redox signaling in promoting $\mathrm{LVH}$. 


\section{Materials and Methods}

\section{Reagents}

The chemiluminescent probe, Bis- $N$-methylacridinium nitrate (lucigenin, catalogue no. 2315-97-1); the reagents L-NAME (N5751), Rotenone (R8875), Oxypurinol (O6881), Diphenyleneiodonium chloride (DPI, D2926), Triton X-100 (T8787) and Tiron (D7389); fluorescein isothiocyanate (FITC)-conjugated wheat germ agglutinin (Lectin, L4895) and; fluorochrome-conjugated anti-goat (C2821) or anti-rabbit (C2306) secondary antibodies were purchased from Sigma-Aldrich, Dorset, UK and gp91 ds-tat (DS-TAT, AS-63818) was bought from AnaSpec, Fremont, USA. NADPH (AC328742500) was purchased from Thermo Fisher Scientific, Waltham, MA, USA. Dihydroethidium (DHE, D11347) was from Invitrogen, Paisley, UK. Polyclonal antibodies targeting Nox2 (sc-20782), Nox4 (sc-30141), phospho-Akt1/2/3 (sc-16646-R), total Akt1/2/3 (sc-8312), total ERK1/2 (sc-292838), total-p38MAPK (sc-535), $\alpha$-tubulin (sc-5546) and the monoclonal antibody targeting phosphop38MAPK (sc-166182) were from Santa Cruz Biotechnology, Dallas, TX, USA. Phospho-ERK1/2 (\#9101) was purchased from Cell Signaling Technology, Danvers, MA, USA.

\section{Animal samples and information}

The cardiac tissue samples used for this study were shared from the animal models [male littermates of C57BL/6J WT and Nox2 knockout (Nox2KO) mice, $n=6$ per group] generated at the University of Surrey and published previously [7]. Animal care and experimentation procedures complied fully with approved standards endorsed in the Guidance on the Operation of the Animals (Scientific Procedures) Act 1986, UK set by the Home Office and the local Animals Ethics Committee of the University of Surrey. At 7 months of age, mice were randomly grouped to be fed either a HFD: $45 \% \mathrm{kcal}$ fat, $20 \% \mathrm{kcal}$ protein and $35 \% \mathrm{kcal}$ carbohydrate (Special Diets Services, Essex, UK), or a normal chow diet (NCD): $13 \%$ kcal fat, $29 \%$ kcal protein and $58 \%$ kcal carbohydrate (LabDiet Ltd, London, UK) for a period of 16 weeks. The following metabolic changes occurred in HFD WT mice after dietary intervention: on average the body weights were increased by $23.4 \%$ in NCD WT mice, $66.9 \%$ in HFD WT mice, $11.6 \%$ in NCD Nox2KO mice and, $41.1 \%$ in HFD Nox2KO mice; blood pressure was increased by $4.8 \%$ in NCD WT mice, $18.7 \%$ in HFD WT mice, decreased by $1.2 \%$ in NCD Nox2KO mice and, increased by $5.7 \%$ in HFD Nox2KO mice. In comparison to NCD controls, heart weights were increased by $14.9 \%$ in HFD WT mice and by $3.5 \%$ in HFD Nox2KO mice and low density lipoprotein levels were increased by a fold increase of 4.6 in HFD WT and by a factor of 8.3 in HFD Nox2KO mice. 


\section{Superoxide $\left(\mathrm{O}_{2}^{\cdot-}\right)$ measurement}

Cardiac LV tissues of C57BL/6J mice in each group (NCD WT, NCD Nox2KO, HFD WT and HFD Nox2KO; $n=6$ per group) were isolated. Disintegrated and homogenised LV tissue was analysed for $\mathrm{O}_{2}{ }^{-}$ generation by means of lucigenin-chemiluminescence (Lumistar, BMG Labtech GmbH, Ortenberg, Germany) using $5 \mu \mathrm{M}$ of lucigenin as previously described [15]. $\mathrm{A} \mathrm{O}_{2}{ }^{--}$scavenger, Tiron $(1 \mathrm{M})$, confirmed $\mathrm{O}_{2}{ }^{--}$generation. Prior to measurement of $\mathrm{O}_{2}{ }^{--}$, the inhibitors targeting nitric oxide synthases (L-NAME, $100 \mu \mathrm{M}$ ), the mitochondrial complex-1 enzymes (Rotenone, $50 \mu \mathrm{M}$ ), xanthine oxidases (Oxypurinol, $250 \mu \mathrm{M}$ ) and flavoproteins (DPI, $20 \mu \mathrm{M}$ or ds-tat, $10 \mu \mathrm{M}$ ) were administered. In situ production of $\mathrm{O}_{2}{ }^{-{ }^{-}}$in intact LV cardiac cells was measured using the redox indicator DHE. Intact LV sections $(6 \mu \mathrm{m})$ were produced using a cryostat (Bright Instruments, Bedfordshire, UK) from tissue ( $3 \times 3 \mathrm{~mm}$ ) obtained from a lower LV region of the harvested mouse hearts. DHE fluorescence was performed as previously described [16]. Within $7 \mathrm{~min}$ of staining, images were captured using the Oxion inverted fluorescent microscope (Euromex, Arnhem, Netherlands) at excitation $518 \mathrm{~nm}$, emission $605 \mathrm{~nm}$. Using HCImageLive (x64) software, fluorescence intensity was quantified, calculated from at least 10 random fields $(254.3 \times 254.3 \mu \mathrm{m})$ for each section, 3 sections per sample and 6 animals per group.

\section{Immunofluorescence staining}

Frozen LV cryosections $(6 \mu \mathrm{m})$ were fixed in a 1:1 methanol:acetone solution and blocked using $2 \%$ BSA with Triton in PBS $1 \mathrm{~h}$ at room temperature inside a dark humidity chamber. Primary antibodies (1:100 dilution) were applied to sections and incubated as mentioned. Polyclonal antibodies targeting: the isoforms Nox1, Nox2 and Nox4; the kinases phospho-Akt1/2/3, total Akt1/2/3, phospho-ERK1/2, total ERK1/2, phospho-p38MAPK, total-p38MAPK and; the loading control $\alpha$-tubulin were used to perform the immunostaining. For the analysis of cardiomyocyte morphology, FITC-conjugated lectin was used. Lectin labeling has been reported to specifically and visibly define single cardiomyocytes for morphological analysis and calculation of myocyte size [17]. Fluorochrome-conjugated anti-goat or anti-rabbit (1:1000 dilution) secondary antibodies were added to the primary antibody-probed sections and incubated as described. Normal rabbit/goat antibody $(5 \mu \mathrm{g} / \mathrm{ml})$ was used as a negative control. Within $12 \mathrm{~h}$ of slide preparation, observation under the Oxion inverted fluorescent microscope was performed (Euromex, Arnhem, Netherlands). The aforementioned methods were used for image capture and quantification. 


\section{Immunoblotting}

Immunoblotting was performed as previously described [18], where protein samples were loaded at a concentration of $40 \mu \mathrm{g}$ per lane. The Biospectrum AC Imaging System (UVP, Cambridge, UK) was used to digitally capture images and to quantify the optical densities of protein bands normalized to loading control bands.

\section{Data and statistical analysis}

All data are presented as mean $(S E M)$. There were 6 mice per group used for the study and experiments were repeated 3 times with a duplicate of each sample per experiment. GraphPad Prism 5 software was used to perform one-way or two-way analysis of variance (ANOVA). Bonferroni post-tests were then performed and significance was accepted at $p<0.05$. 


\section{Results}

\section{Nox2KO improves excessive cardiac $\mathrm{O}_{2}{ }^{*-}$ generation from chronic $\mathrm{HFD}$ feeding}

NADPH-dependent $\mathrm{O}_{2}{ }^{\cdot-}$ production was significantly higher for HFD WT hearts than corresponding WT controls (Figure 1a and b). Tiron addition, a cell membrane permeable $\mathrm{O}_{2}{ }^{--}$scavenger, significantly reduced the signal, verifying $\mathrm{O}_{2}^{--}$measurement. Contrastingly, there were significantly lower levels of NADPHdependent $\mathrm{O}_{2}{ }^{--}$production in Nox2KO hearts compared to HFD WT values (Figure 1b). Inhibition significantly reduced $\mathrm{O}_{2}{ }^{--}$levels by Tiron, DPI (an inhibitor of flavoproteins) and DS-TAT (an inhibitor of Nox assembly) with no significant difference observed under the influence of L-NAME (an inhibitor of nitric oxide synthases), Rotenone (an inhibitor of mitochondrial complex 1 enzymes) nor Oxypurinol (an inhibitor of xanthine oxide synthases) (Figure 1c). In situ intensities of tiron-inhibitable $\mathrm{O}_{2}{ }^{--}$generation was significantly greater in HFD WT LV sections, as opposed to their NCD WT controls, and was significantly dampened in HFD Nox2KO LV sections compared to HFD WT values (Figure 1d). 
Figure 1.
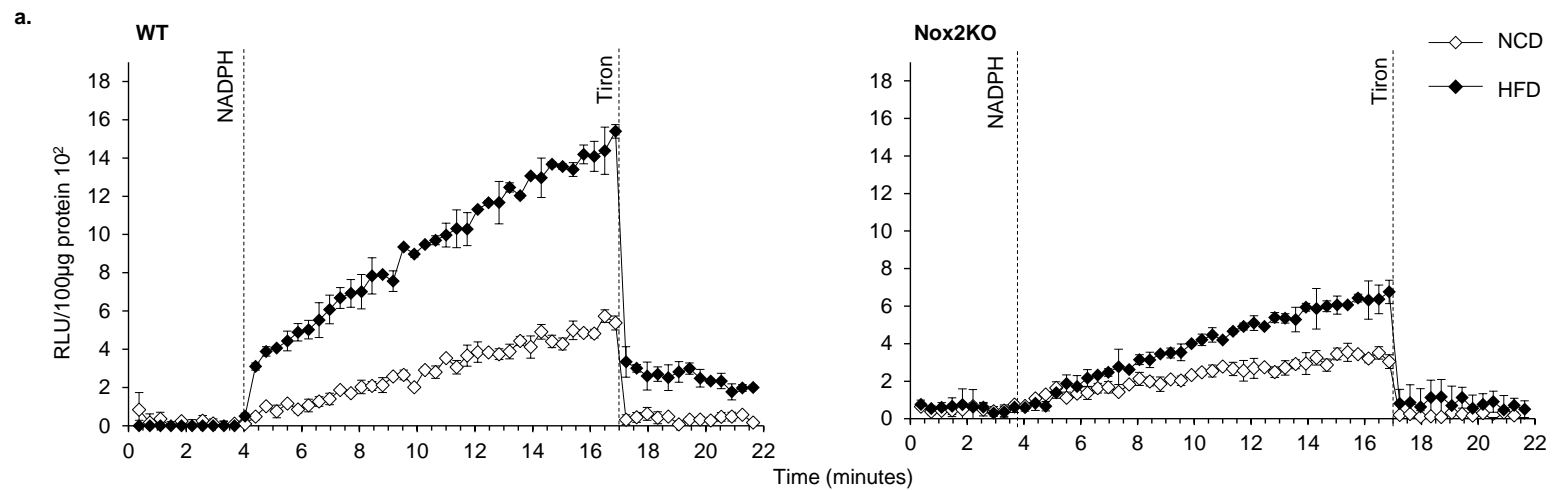

b.

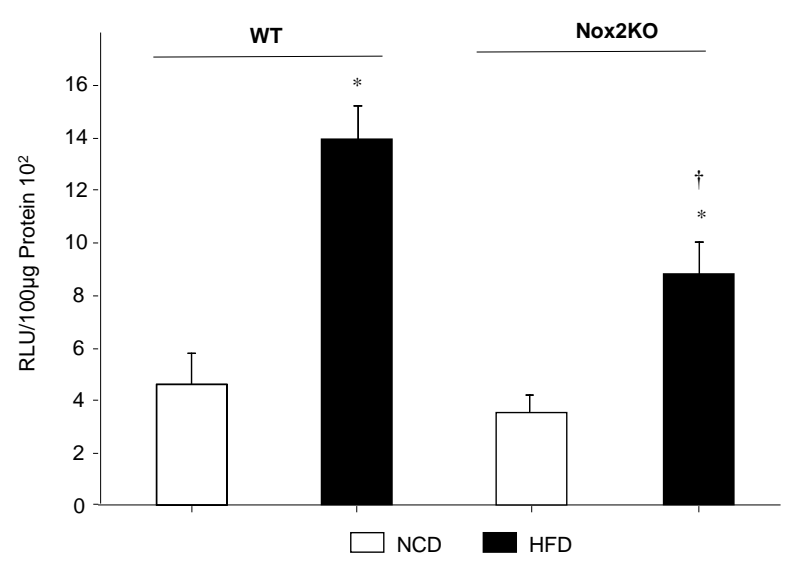

HFD WT cardiac homogenates

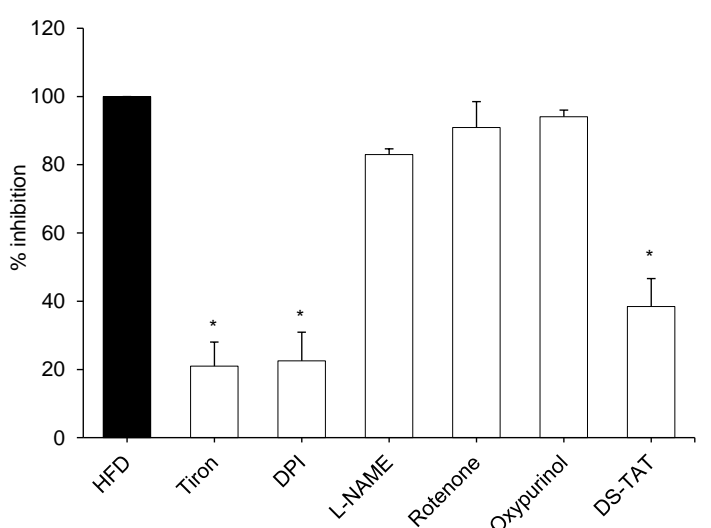

d.
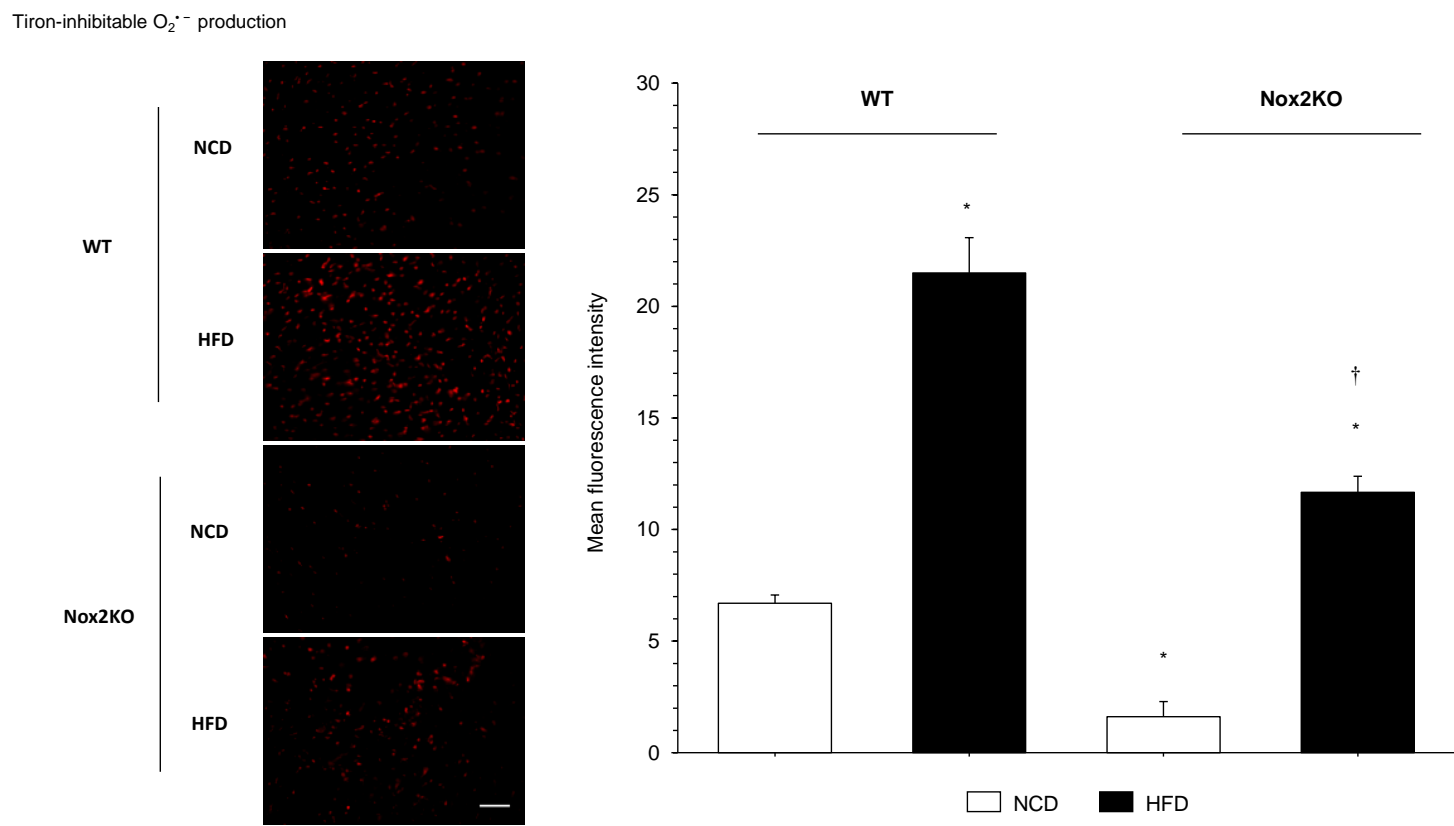

Figure 1. Superoxide $\left(\mathrm{O}_{2}{ }^{\circ}\right)$ generation detected by dual methodology in wild-type (WT) and Nox2 knockout (Nox2KO) hearts after high fat diet (HFD) intervention. Lucigenin-chemiluminescence was used to acquire: (a) representative kinetic measurements of $\mathrm{O}_{2}{ }^{-}$production in relative light units (RLU) where NADPH substrate was added at $4 \mathrm{~min}$ and Tiron at $17 \mathrm{~min}$ (dashed lines) and; (b) bar figure representing $\mathrm{O}_{2}^{\circ}$ production. (c) Lucigenin-chemiluminescence detection of enzyme inhibition of $\mathrm{O}_{2}^{-}$in HFD WT hearts. DPI, diphenylene iodonium. $* p<0.05$, significantly different from HFD values. (d) Dihydroethidium (DHE) fluorescence detection 
of representative in situ $\mathrm{O}_{2}{ }^{\circ-}$ generation (left panel). Scale bar $=50 \mu \mathrm{m}$. Fluorescence intensity was quantified for tiron-inhibitable production (right panel). Fluorescence intensity was calculated from a minimum of 10 random fields $(254.3 \times 254.3 \mu \mathrm{m})$ per 3 sections in each group. ${ }^{*} p<0.05$, significantly different from normal chow diet (NCD) WT values; $\dagger p<0.05$, significantly different from HFD WT values. $n=6$ mice per group.

\section{HFD-induced expression of NADPH Oxidases}

HFD WT hearts had significantly higher levels of Nox2 immunoblot protein expression, but not Nox1 or Nox4 compared to NCD WT controls (Figure 2a). Confirming the Nox2KO, NCD and HFD Nox2KO hearts had significantly reduced Nox2 expression levels compared to HFD WT hearts. Nox4 was significantly increased in both NCD and HFD Nox2KO hearts (Figure 2a, right lower panel) which was not observed for Nox1, suggesting a compensatory upregulation of Nox4 in the absence of Nox2 regardless of HFD-intervention. Nox2 immunofluorescence staining levels were significantly higher in HFD WT hearts than NCD WT controls (Figure 2b) and mirrored the result of negligible levels of Nox2 expression in Nox2KO samples. 
Figure 2.

a. Nox expression detected by Western blots
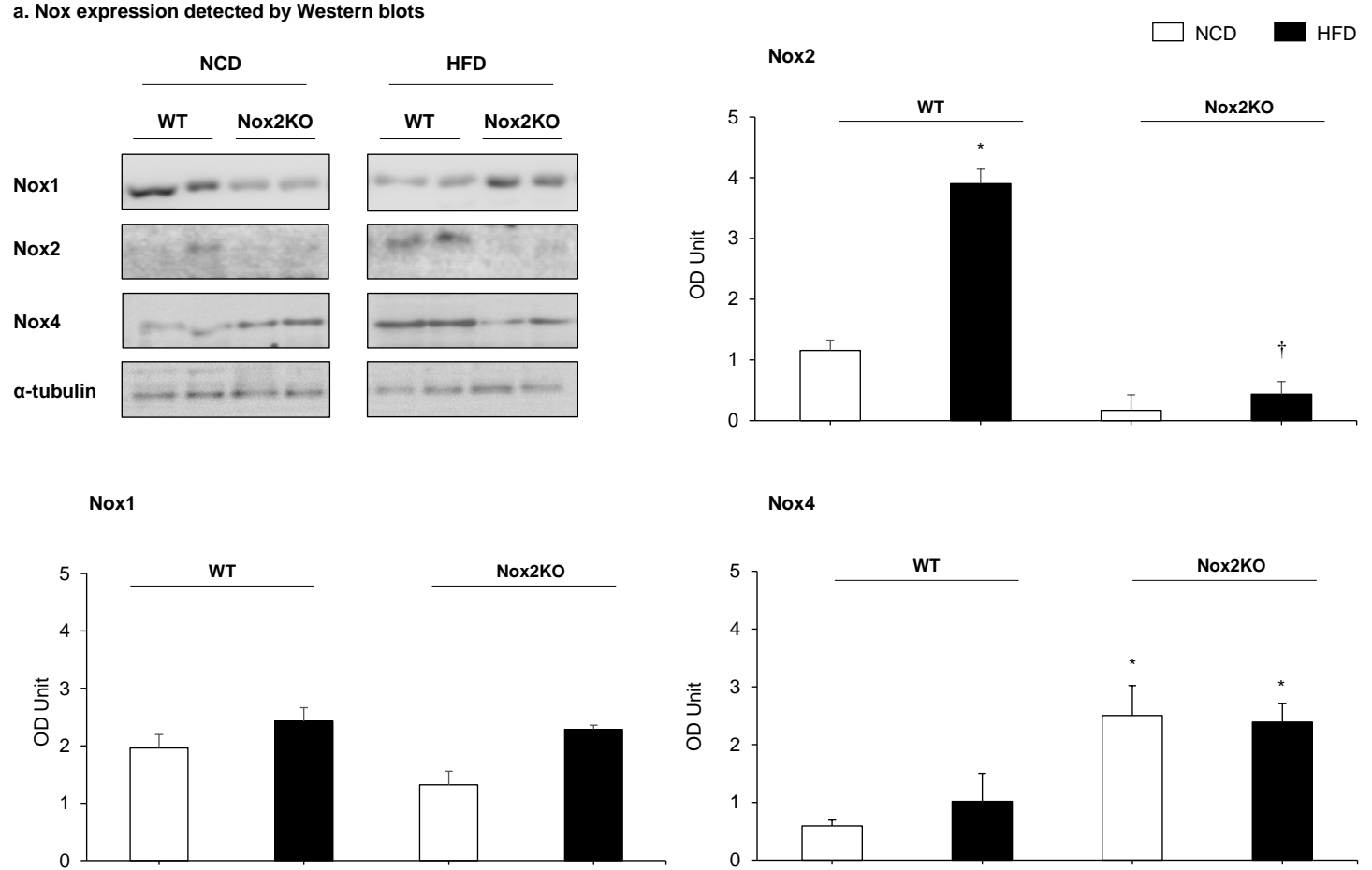

b. Immunofluorescence detection of Nox2 expression
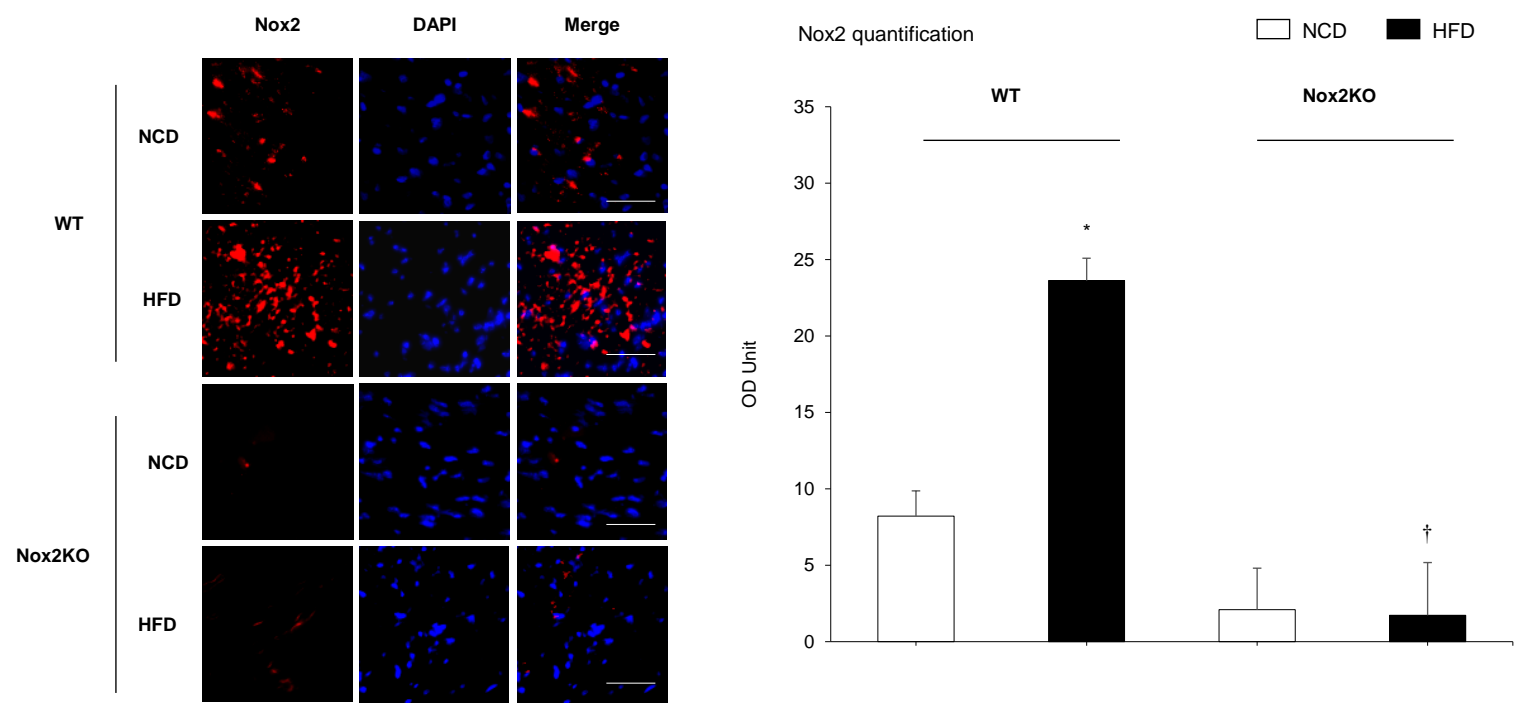

Figure 2. Left ventricular Nox protein expression subject to normal chow diet (NCD) and high-fat diet (HFD). (a) Western blot analysis confirming expression levels in wild-type (WT) and Nox2 knockout (Nox2KO) hearts in NCD (left panel) and HFD (right panel) conditions. The loading control used was $\alpha$-tubulin. Bar represents the mean (SEM) of digitally quantified optical densities (OD) of gel bands normalized to $\alpha$-tubulin levels. Representative gel bands are shown (upper left panel). (b) Representative immunofluorescence staining using gp91 phox antibody targeting Nox2 (red) and DAPI for nuclear staining (blue). Rightmost panel represents merged composites. The fluorescence intensity of individual molecule was quantified to generate the quantification bar figure from 10 images at X40 magnification captured from 3 sections of $6 \mu \mathrm{m}$ thickness per group (right panel). Scale bar $=50 \mu \mathrm{m}$. Data are presented as mean $(S E M) .{ }^{*} p<0.05$, significantly different from corresponding NCD values, ${ }^{\dagger} p<0.05$, significantly different from WT HFD values. $n=6$ mice per group. 


\section{Nox2-mediated altered redox signaling in the establishment of left ventricular hypertrophy}

The cross-sectional area (CSA) of lectin-stained left ventricular (LV) morphology was significantly greater in HFD WT hearts as compared to NCD controls, which was reduced significantly in Nox2KO hearts (Figure 3a), suggesting a role of Nox2 in the hypertrophic response. Pro-hypertrophic kinase expression levels of p-Akt and p-ERK1/2 were significantly increased in the immunostaining of HFD WT sections compared to NCD controls whereas this activation of Akt and ERK1/2 was significantly rescued to NCD levels in Nox2KO sections, indicating a role of Nox 2 in the stimulation of Akt and ERK1/2 under dietary induced conditions (Figure $3 \mathrm{~b}$ and c). Strengthening these findings, p-Akt and p-ERK1/2 expression by Western blot analysis were significantly increased in HFD WT hearts compared with NCD WT values, $p<0.05$ (Figure $4 \mathrm{a}, \mathrm{b}$ and c). In HFD-fed Nox2KO hearts however, this expression was significantly reduced for both p-Akt and p-ERK1/2 (Figure $4 \mathrm{~b}$ and c). P-p38MAPK levels rose significantly in HFD Nox2KO hearts in comparison to NCD Nox2KO controls, $p<0.05$ (Figure 4d). However, the increase in the levels of p-p38MAPK in HFD WT hearts was not found to be significant. 
Figure 3.

a. Representative LV cross-sections and cardiomyocyte sizes
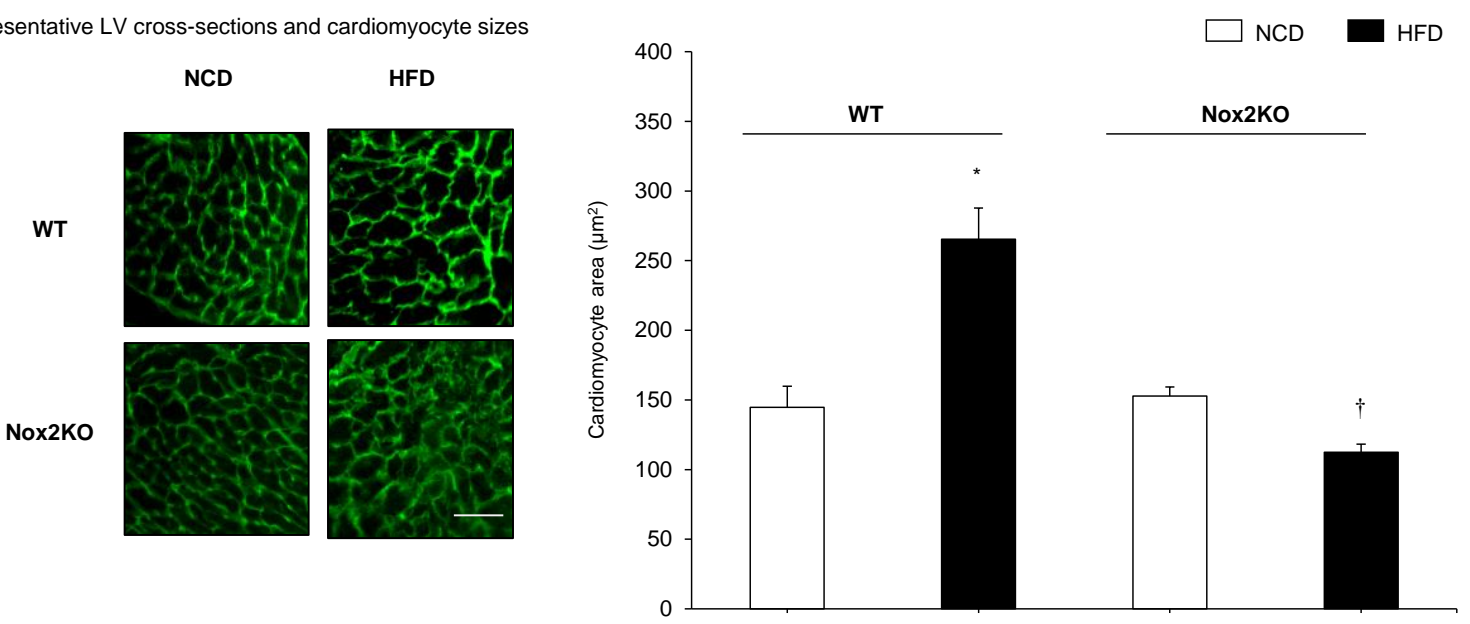

b. Immunofluorescence detection of P-Akt expression
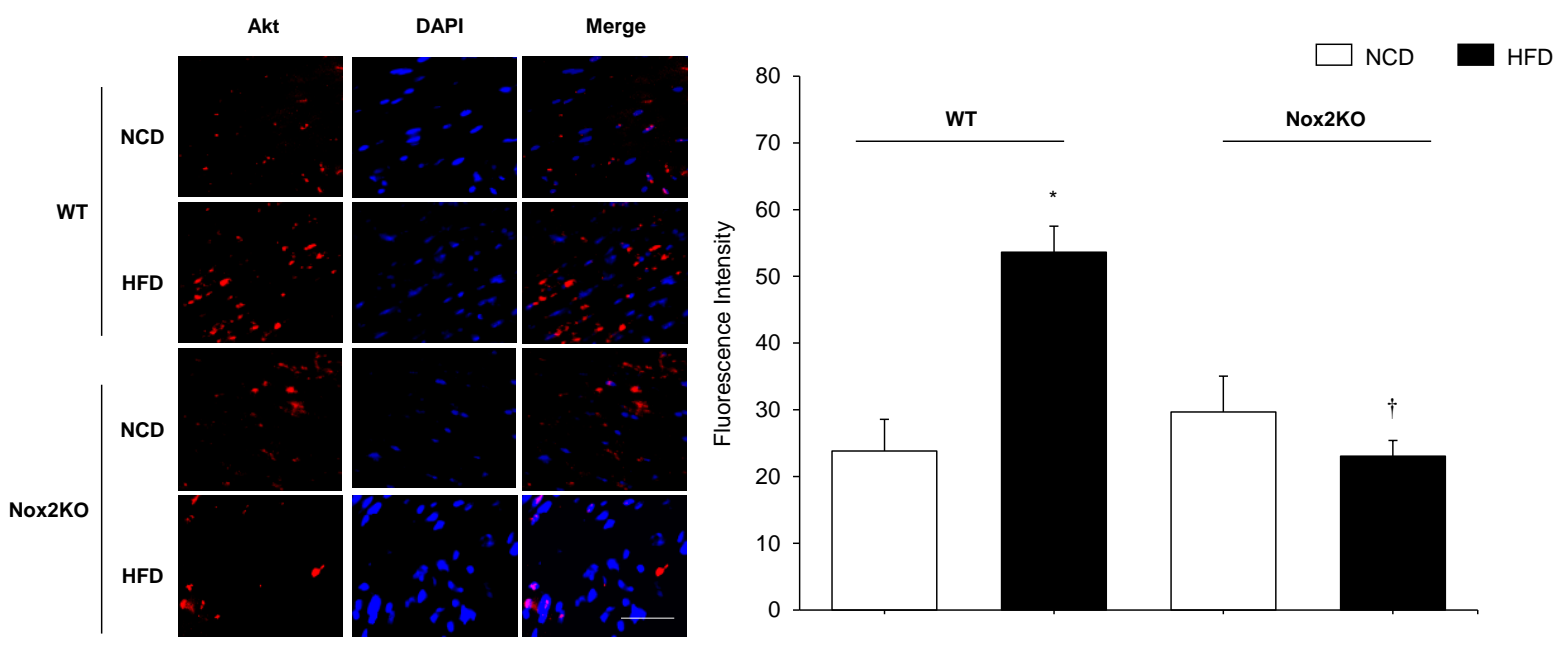

c. Immunofluorescence detection of P-ERK1/2 expression
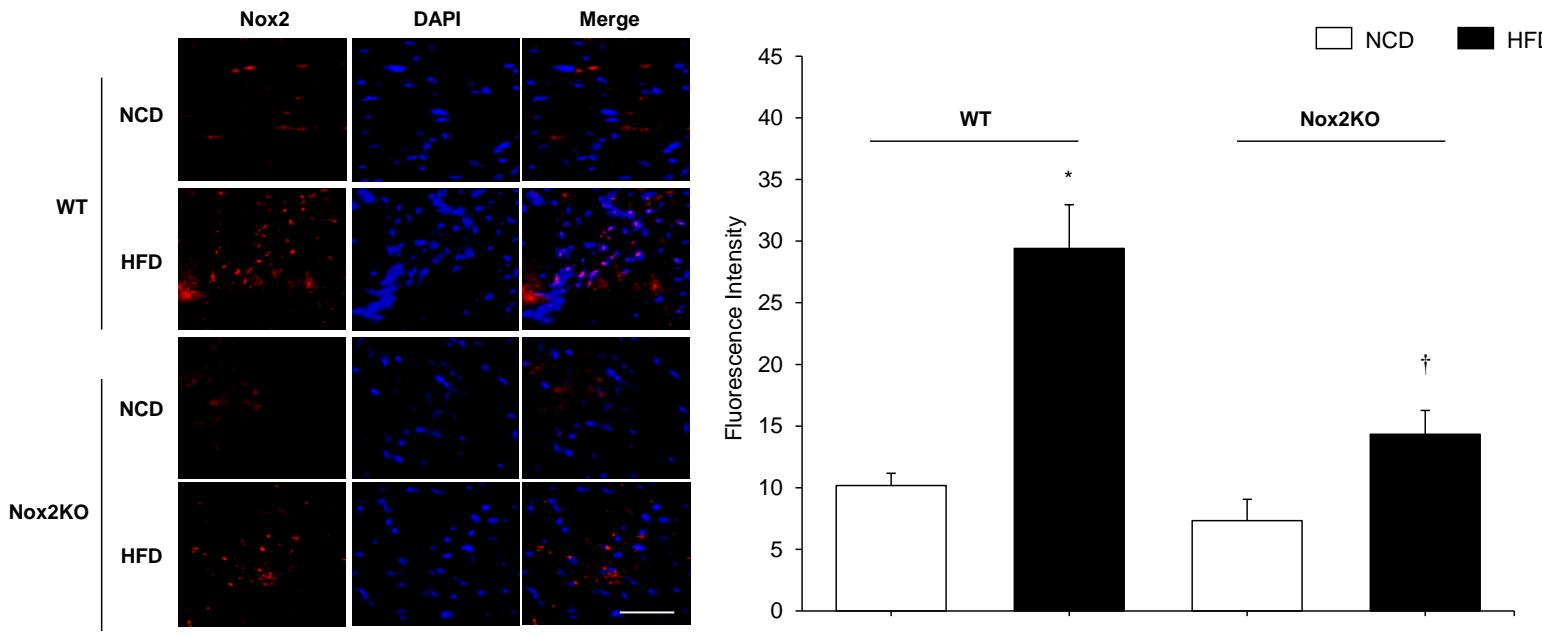

Figure 3. Cardiomyocyte size and immunofluorescence staining of phosphorylated Akt 1/2/3 (P-Akt 1/2/3) and ERK1/2 (P-ERK1/2) on left ventricular (LV) cryosections. (a) Cardiomyocyte membrane visualization by immunofluorescence staining using FITC-conjugated wheat germ agglutinin on LV sections (left panel). The cross-sectional area $\left(\mu \mathrm{m}^{2}\right)$ was quantified and calculated using 10 random fields taken from 3 sections per sample (right panel). Representative visualization of sections using: (b) P-Akt1/2/3 antibody (red) and; (c) P-ERK1/2 
antibody (red) with DAPI for nuclear staining (blue). Rightmost lane represents merged composites. The fluorescence intensity of individual molecules was quantified to generate the quantification bar figure from 10 images at X40 magnification captured from 3 sections of $6 \mu \mathrm{m}$ thickness per group (right panel). Scale bar $=50$ $\mu \mathrm{m} .{ }^{*} p<0.05$, significantly different from corresponding normal chow diet (NCD) values. ${ }^{\dagger} p<0.05$, significantly different from corresponding wild-type (WT) values. One-way ANOVA. $n=6$ mice per group.

Figure 4.
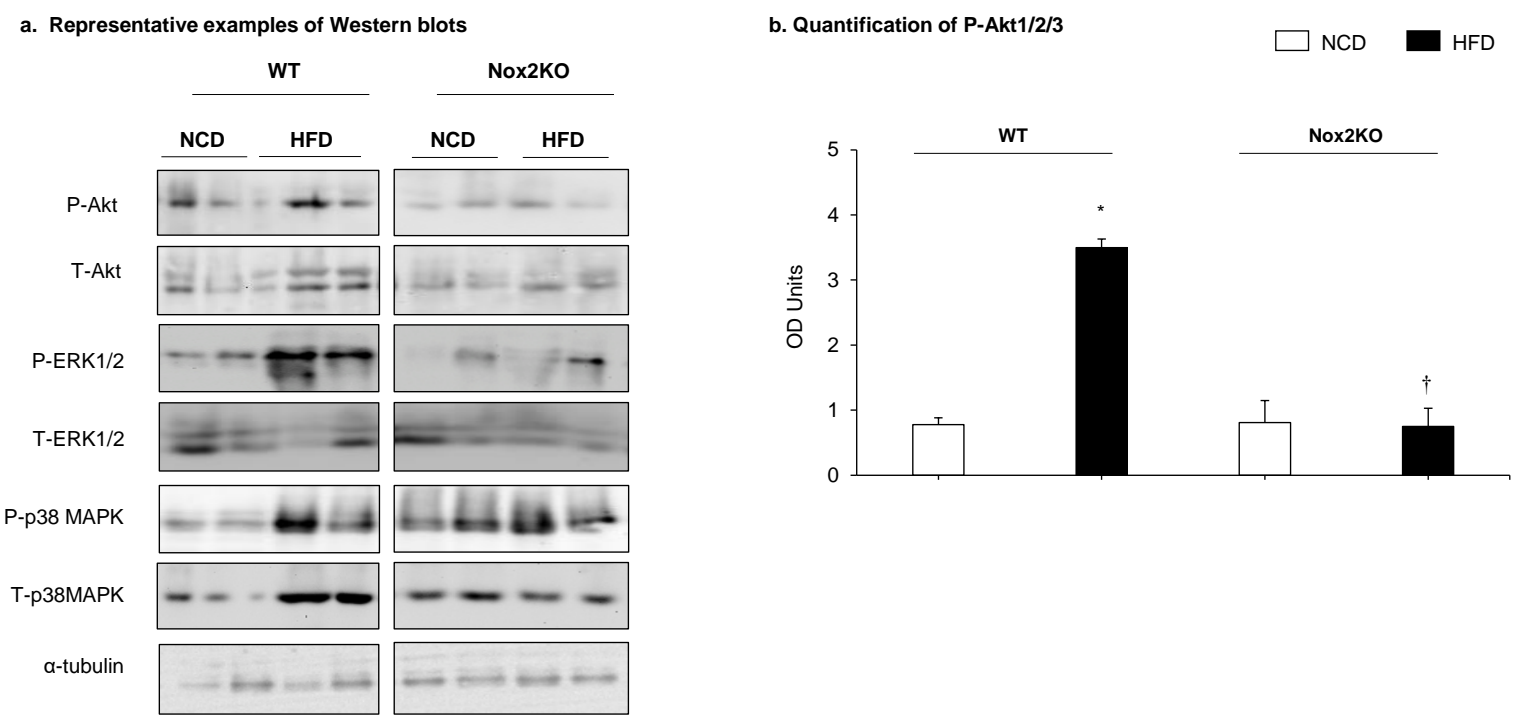

c. Quantification of P-ERK1/2

d. Quantification of P-p38MAPK
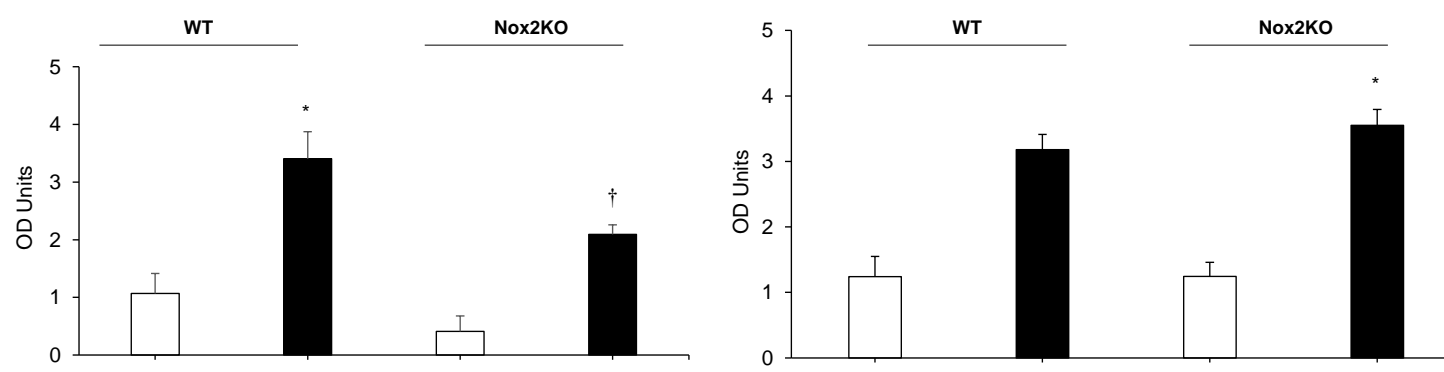

Figure 4. Western blot detection of Akt, ERK1/2 and p38MAPK phosphorylation in cardiac homogenates administered different diets. (a) Representative kinase expression levels in WT (left panel) and Nox2KO (right panel) hearts under non-chow diet (NCD) or high-fat diet (HFD) conditions. The loading control used was $\alpha$ tubulin. Bar represent the mean (SEM) of digitally quantified optical densities (OD) of gel bands normalized to $\alpha$ tubulin levels of the kinases: (b) Akt1/2/3; (c) ERK1/2 and; (d) p38MAPK. Activated forms of the protein phospho-Akt (P-Akt), phospho-ERK1/2 (P-ERK1/2) and phospho-p38MAPK (P-p38MAPK) were quantified relative to total protein (T-Akt, T-ERK1/2 and T-p38MAPK, respectively). ${ }^{*} p<0.05$, significantly different from NCD values, ${ }^{\dagger} p<0.05$, significantly different from corresponding HFD values. One-way ANOVA. $n=6$ mice per group. 


\section{Discussion}

Presently, Nox2 is acknowledged as a key influence in global metabolic oxidative disorder and cardiovascular dysfunction [4]. Herein, the novel findings are that a HFD evokes: (1) excessive Nox2-mediated cardiac oxidative stress to endorse LVH; (2) increased Nox2-dependent activation of Akt, ERK1/2, but not p38MAPK in myocardium. For the first time it is reported that the growth regulating kinases Akt and ERK1/2 are found to be partly Nox2-driven upon lipid stimulation to promote cardiac hypertrophy in middle-aged mice. However, despite evidence of pro-hypertrophic roles of the kinase p38MAPK [19], under HFD this study failed to observe its activation.

Individuals consuming westernised diets have greater prevalence of concentric LVH [20]. At the stages of obesity, pressure-overload establishes cardiomyocyte enlargement, increases in ventricular dimensions, fibrosis and dysfunction [21]. It is acknowledged that the intracellular mechanisms by which lipid-stimulated cardiac hypertrophy occurs remains incompletely understood [22]. This study has provided insight of the prohypertrophic influence of cardiac Nox 2 in the alteration of cardiomyocyte morphology in the mouse model of HFD-induced obesity. It is known that Nox2-derived ROS induces phenotypic changes such as endothelial dysfunction, interstitial fibrosis, myocardial contractility and myocyte hypertrophy [7]. However, others report LVH in response to pressure-overload did not require the action of Nox 2 though other cardiac abnormal events such as fibrosis appeared to be Nox2-dependent [23]. Nox2-derived ROS is shown to be important for cardiac hypertrophy in response to angiotensin II [24], after myocardial infarction [23] and upon doxorubicin treatment [25]. It has been postulated that Nox 2 mediates stimuli-specific roles in the promotion of hypertrophy and the investigated pathway in the HFD-fed heart appears to be limited and controversial [23]. We introduce fresh insight that Nox2 may be stimulated by lipid induction in the establishment of cardiomyocyte hypertrophy.

It has been proposed that lipid activation of protein synthesis and growth-stimulating genes have significant influence in the establishment of LVH [26]. A novel indication revealed herein is that HFD promotes Nox2-mediated activation of pro-hypertrophic kinases Akt and ERK1/2 in myocardium. We find that the results of increased HFD-induced p-Akt expression in the heart falls in line with a report in myocardium [27], though this study did not investigate the contribution of Nox2. Interestingly, diminished levels of HFD-induced activated Akt are reported in skeletal muscle and aorta resulting in the impairment of insulin sensitivity after HFD feeding [6,7], suggestive of tissue-type specific signaling mediated by HFD-induced Nox 2 activation. The observation of increased cardiomyocyte p-Akt promoting impaired insulin signaling is suggested to induce 
insulin resistance via mechanisms relating to the dysregulated activation of GLUT4 transporters despite GLUT4 translocation remaining intact [22]. Moreover, it has long been suggested that there is an association between insulin sensitivity and LV enlargement [28]. P-Akt-mediated development of cardiac hypertrophy in obesogenic environments is thought to be mediated via the inhibition of the transcription factor Foxo3a [29]. Therefore, increased Akt activation by Nox 2 may augment its established dual roles in the activation of programs involved in both insulin signaling and cardiomyocyte hypertrophy. Further investigation, beyond that of excessive HFDinduced Akt activation observed herein, is required to understand the HFD-induced insulin response in the heart [22].

Nox2-derived ROS production caused by excessive glucose and insulin challenge also elicits ERK1/2 activation, resulting in endothelial cell injury, dysfunction and death [30]. Studies report that Nox2 augments endothelial p-ERK1/2 expression after chronic HFD feeding [7,11]. Our findings strongly suggest a contribution of myocardial Nox2 in HFD-induced ERK1/2 activation. The results also suggest that Nox2 may not have a role in myocardial activation of p38MAPK. The role of the Nox2 isoform in stimulating p38MAPK activation is not broadly researched however as fibrotic events causing cardiac remodelling are induced by HFD in vivo [31], the role of Nox2-derived $\mathrm{O}_{2}{ }^{--}$on the activation of the pro-fibrotic p38MAPK in the heart may therefore be beneficial to better understand the causes of cardiac remodelling in HFD conditions.

Reports had implicated Nox2 as an instrumental driver of CVD pathogenesis and topical interest of selectively targeting Nox2 as therapeutic strategy has broadened [32]. Global targeting of Nox2 however may be unsuitable as evidence indicates this could evoke enhanced susceptibility to infection [33]. Therefore, we suggest cardiac-specific targeting of Nox2 activation may be a viable approach to preserving myocardial function in metabolic conditions.

\section{Conclusions}

In summary, the current study has provided the novel insight that HFD-induced obesity in middle-aged mice is associated with Nox2-mediated cardiac oxidative stress, altered redox signaling events and cardiomyocyte expansion. Cardiac-specific targeting of Nox 2 may promote a viable therapy to reduce metabolic-related oxidative stress in the heart, redox signaling derangements and protect against LVH. 


\section{Author contributions}

SNB: gathered experimental data, performed statistical analysis, and drafted the manuscript; JML: provided the samples, supervised the study and critically reviewed the manuscript. All authors have read and approved the final version of the manuscript, and agree with the order of presentation of authors.

\section{Funding}

This research was funded by the British Heart Foundation (grant number: PG/14/85/3116).

\section{Declaration of competing interest}

The authors declare that they have no competing interests

\section{Acknowledgements}

Animal care and experimentation procedures were conducted in accordance with the protocols verified by the Home Office under the Animals (Scientific Procedures) Act 1986, UK and the local Animal Ethics Committee of the University of Surrey.

\section{References}

[1] W. Dhahri, M.C. Drolet, E. Roussel, et al. Chronic high-fat diet-induced obesity decreased survival and increased hypertrophy of rats with experimental eccentric hypertrophy from chronic aortic regurgitation, BMC Cardiovasc. Disord. 14 (2014). https://doi.org/10.1186/1471-2261-14-123.

[2] J. Bentham, M. Di Cesare, V. Bilano, et al. Worldwide trends in body-mass index, underweight, overweight, and obesity from 1975 to 2016: a pooled analysis of 2416 population-based measurement studies in 128.9 million children, adolescents, and adults, Lancet. 390 (2017) 2627-2642. https://doi.org/10.1016/S0140-6736(17)32129-3.

[3] A.M. Lehnen, B. Rodrigues, M.C. Irigoyen, et al. Cardiovascular Changes in Animal Models of Metabolic Syndrome, J. Diabetes Res. 2013 (2013) 11. https://doi.org/10.1155/2013/761314.

[4] D. Burtenshaw, R. Hakimjavadi, E.M. Redmond, et al. Nox, Reactive Oxygen Species and Regulation of Vascular Cell Fate, Antioxidants 6 (2017) 90. https://doi.org/10.3390/antiox6040090.

[5] A. Sirker, M. Zhang, A.M. Shah, NADPH oxidases in cardiovascular disease: Insights from in vivo models and clinical studies, Basic Res. Cardiol. 106 (2011) 735-747. https://doi.org/10.1007/s00395- 
011-0190-z.

[6] A.S.P. De Figueiredo, A.B. Salmon, F. Bruno, et al. Nox2 mediates skeletal muscle insulin resistance induced by a high fat diet, J. Biol. Chem. 290 (2015) 13427-13439.

https://doi.org/10.1074/jbc.M114.626077.

[7] J. Du, L.M. Fan, A. Mai, et al. Crucial roles of Nox2-derived oxidative stress in deteriorating the function of insulin receptors and endothelium in dietary obesity of middle-aged mice, Br. J. Pharmacol. 170 (2013) 1064-1077. https://doi.org/10.1111/bph.12336.

[8] Z. Liu, I.Y. Patil, T. Jiang, et al. High-Fat Diet Induces Hepatic Insulin Resistance and Impairment of Synaptic Plasticity, PLoS One. 10 (2015) e0128274. https://doi.org/10.1371/journal.pone.0128274.

[9] R.L. Adochio, J.W. Leitner, K. Gray, et al. Early responses of insulin signaling to high-carbohydrate and high-fat overfeeding, Nutr. Metab. 6 (2009) 37. https://doi.org/10.1186/1743-7075-6-37.

[10] B.A. Rose, T. Force, Y. Wang, Mitogen-Activated Protein Kinase Signaling in the Heart: Angels Versus Demons in a Heart-Breaking Tale, (2010). https://doi.org/10.1152/physrev.00054.2009.

[11] A. Mai, J.-M. Li, NADPH oxidase activation and oxidative stress in high-fat diet-induced hypertension and metabolic disorders, Heart. 100 (2014) A1.3-A1. https://doi.org/10.1136/heartjnl-2014-306916.3.

[12] J. Ketonen, T. Pilvi, E. Mervaala, Caloric restriction reverses high-fat diet-induced endothelial dysfunction and vascular superoxide production in C57B1/6 mice, Heart Vessels. 25 (2010) 254-262. https://doi.org/10.1007/s00380-009-1182-x.

[13] A. Sirker, C.E. Murdoch, A. Protti, et al. Cell-specific effects of Nox2 on the acute and chronic response to myocardial infarction, J. Mol. Cell. Cardiol. 98 (2016) 11-17. https://doi.org/10.1016/j.yjmcc.2016.07.003.

[14] J.M. Li, N.P. Gall, D.J. Grieve, et al. Activation of NADPH oxidase during progression of cardiac hypertrophy to failure, Hypertension. 40 (2002) 477-484. https://doi.org/10.1161/01.HYP.0000032031.30374.32.

[15] L. Teng, L.M. Fan, D. Meijles, et al. Divergent effects of p47(phox) phosphorylation at S303-4 or S379 on tumor necrosis factor- $\alpha$ signaling via TRAF4 and MAPK in endothelial cells. Arterioscler. Thromb. Vasc. Biol. 32 (2012) 1488-96. https://doi.org/10.1161/ATVBAHA.112.247775.

[16] J.-M. Li, S. Wheatcroft, L.M. Fan, et al. Opposing Roles of p47 phox in Basal Versus Angiotensin IIStimulated Alterations in Vascular $\mathrm{O}_{2}{ }^{-}$Production, Vascular Tone, and Mitogen-Activated Protein Kinase Activation, Circulation. 109 (2004) 1307-1313. 
https://doi.org/10.1161/01.CIR.0000118463.23388.B9.

[17] A. D'souza, F.C. Howarth, J. Yanni, et al. Chronic effects of mild hyperglycaemia on left ventricle transcriptional profile and structural remodelling in the spontaneously type 2 diabetic Goto-Kakizaki rat, Heart Failure Reviews (2014) 65-74. https://doi.org/10.1007/s10741-013-9376-9.

[18] S. Thakur, J. Du, S. Hourani, et al. Inactivation of adenosine A2A receptor attenuates basal and angiotensin II-induced ROS production by Nox2 in endothelial cells, J. Biol. Chem. 285 (2010) 4010440113. https://doi.org/10.1074/jbc.M110.184606.

[19] M.S. Marber, B. Rose, Y. Wang, The p38 mitogen-activated protein kinase pathway-A potential target for intervention in infarction, hypertrophy, and heart failure, J. Mol. Cell. Cardiol. 51 (2011) 485-490. https://doi.org/10.1016/j.yjmcc.2010.10.021.

[20] A. Maugeri, J. Hruskova, J. Jakubik, et al. How dietary patterns affect left ventricular structure, function and remodelling: evidence from the Kardiovize Brno 2030 study, Sci. Rep. 9 (2019) 1-10. https://doi.org/10.1038/s41598-019-55529-5.

[21] B. Debosch, I. Treskov, T.S. Lupu, et al. Akt1 Is Required for Physiological Cardiac Growth, (2006). https://doi.org/10.1161/CIRCULATIONAHA.105.595231.

[22] Y. Zhu, R.O. Pereira, B.T. O’Neill, et al. Cardiac PI3K-Akt Impairs Insulin-Stimulated Glucose Uptake Independent of mTORC1 and GLUT4 Translocation, Mol. Endocrinol. 27 (2013) 172-184. https://doi.org/10.1210/me.2012-1210.

[23] Y.H. Looi, D.J. Grieve, A. Siva, et al. Involvement of Nox2 NADPH Oxidase in Adverse Cardiac Remodeling After Myocardial Infarction, (2008). https://doi.org/10.1161/HYPERTENSIONAHA.107.101980.

[24] M. Satoh, H. Ogita, K. Takeshita, et al. Requirement of Rac1 in the development of cardiac hypertrophy, Proc. Natl. Acad. Sci. U. S. A. 103 (2006) 7432-7437. https://doi.org/10.1073/pnas.0510444103.

[25] Y. Zhao, D. McLaughlin, E. Robinson, et al. Nox2 NADPH oxidase promotes pathologic cardiac remodeling associated with doxorubicin chemotherapy, Cancer Res. 70 (2010) 9287-9297. https://doi.org/10.1158/0008-5472.CAN-10-2664.

[26] B.N. Finck, X. Han, M. Courtois, et al. A critical role for PPAR $\alpha$-mediated lipotoxicity in the pathogenesis of diabetic cardiomyopathy: Modulation by dietary fat content, Proc. Natl. Acad. Sci. U. S. A. 100 (2003) 1226-1231. https://doi.org/10.1073/pnas.0336724100. 
[27] A. Jovanovic, E. Sudar-Milovanovic, M. Obradovic, et al. Influence of a High-Fat Diet on Cardiac iNOS in Female Rats, Curr. Vasc. Pharmacol. 15 (2016) 491-500. https://doi.org/10.2174/1570161114666161025101303.

[28] R.A. Phillips, L.R. Krakoff, A. Dunaif, et al. Relation among Left Ventricular Mass, Insulin Resistance, and Blood Pressure in Nonobese Subjects ${ }^{1}$, J. Clin. Endocrinol. Metab. 83 (1998) 4284-4288. https://doi.org/10.1210/jcem.83.12.5331.

[29] C.X. Fang, F. Dong, D.P. Thomas, et al. Hypertrophic cardiomyopathy in high-fat diet-induced obesity: Role of suppression of forkhead transcription factor and atrophy gene transcription, Am. J. Physiol. Hear. Circ. Physiol. 295 (2008) H1206. https://doi.org/10.1152/ajpheart.00319.2008.

[30] L.M. Fan, S. Cahill-Smith, L. Geng, et al. Aging-associated metabolic disorder induces Nox2 activation and oxidative damage of endothelial function, Free Radic. Biol. Med. 108 (2017) 940-951. https://doi.org/10.1016/j.freeradbiomed.2017.05.008.

[31] F. Martins, D.H.S. Campos, L.U. Pagan, et al. Dieta hiperlipídica promove remodelação cardíaca em modelo experimental de obesidade, Arq. Bras. Cardiol. 105 (2015) 479-486. https://doi.org/10.5935/abc.20150095.

[32] J. Streeter, W. Thiel, K. Brieger, et al. Opportunity Nox: The Future of NADPH Oxidases as Therapeutic Targets in Cardiovascular Disease, Cardiovasc. Ther. 31 (2013) 125-137. https://doi.org/10.1111/j.1755-5922.2011.00310.x.

[33] S.P. Gray, E. Di Marco, J. Okabe, et al. NADPH Oxidase 1 plays a key role in diabetes mellitusaccelerated atherosclerosis, Circulation. 127 (2013) 1888-1902. https://doi.org/10.1161/CIRCULATIONAHA.112.132159. 